\title{
DNA Repairing and its Mechanism in the Cell
}

\author{
Manu Mitra* \\ Department of Electrical Engineering, Alumnus of University of Bridgeport, Bridgeport, USA
}

*Corresponding Author: Manu Mitra, Department of Electrical Engineering, Alumnus of University of Bridgeport, Bridgeport, USA.

Received: June 18, 2019; Published: July 16, 2019

DOI: 10.31080/ASMS.2019.03.0356

\begin{abstract}
DNA (Deoxyribonucleic Acid) repair denotes collection of processes by which a cell identifies and corrects the damage to the DNA molecules that encode its genome. Several incisions causes structural damage to the DNA molecule and may alter or eliminate the cell's ability to transcribe the gene that are affected DNA encodes.

There are many techniques and methods to repair DNA, however, in this paper few methods are reviewed. For instance - Mechanism for repairing damaged DNA, Novel technique to repair damaged DNA, Scientist confirm DNA repair, Repairing faulty genes to cure diseases, Repairing DNA: Structure of Mre11 protein bound.

Keywords: DNA; DNA Repair; Genome; Genes
\end{abstract}

\section{Introduction}

DNA (Deoxyribonucleic Acid) gets damaged all the time not just during replication but also by outside factors such as UV lights, chemicals, X-rays and not to mention spontaneous chemical reactions that occur even without environmental changes. Providentially cells have repair mechanisms to find and correct many types of DNA damage. Repair process include but not limited to 1) direct reversal - DNA damaging reactions can be directly "fixed" by enzymes in the cell. 2) Excision Repair - damage to one or a few bases of DNA can directly undone and can be replaced the damaged region. In base excision repair, just damaged base is removed. In nucleotide excision repair, nucleotides is removed. 3) Double stranded break repair - Two major ways, non-homologous end joining and homologous recombination are used to repair double stranded breaks in DNA (when an entire chromosome splits into two pieces) [1].

\section{Mechanism for repairing damaged DNA}

Homologous recombination (HR) is a technique that repairs damaged DNA with perfect accuracy, it uses homologous sequence from another DNA as a template. This process involves getting together of 2 DNA molecules, an examination for homologous sequences and exchange of DNA strands.

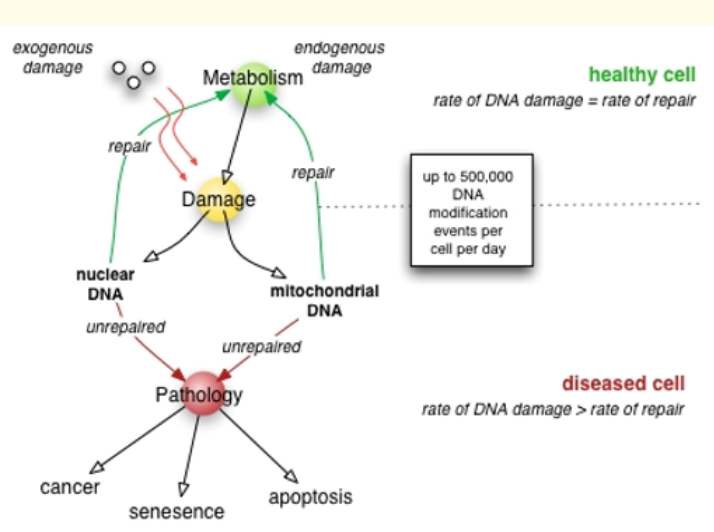

Figure 1: Illustrates DNA repair rate is an crucial and important determinant of cell pathology [2].

RecA family proteins are the major and central recombination's for Homologous recombination. The family consist of prokaryotic RecA, archaeal RadA, eukaryotic Rad51 and Dmc1. They are vital roles in genome maintenance, cell proliferation and more importantly, in higher eukaryotes. For instance Rad51-deficient vertebrate cells collect chromosomal breaks before death. Rad51 and its meiosis-specific homolog. Dmc1 are also essential for meiosis 
a specialized cell cycle for production of gametes. Mammalian Rad51 and Dmc1 proteins are known to work together with tumor suppressor proteins such as BRCA2.

This research was led by the team by Dr. Andrew H. -J. Wang and Dr. Ting-Fang Wang at the Institute of Biological Chemistry, Academia Sinica (IBCAS) [3].

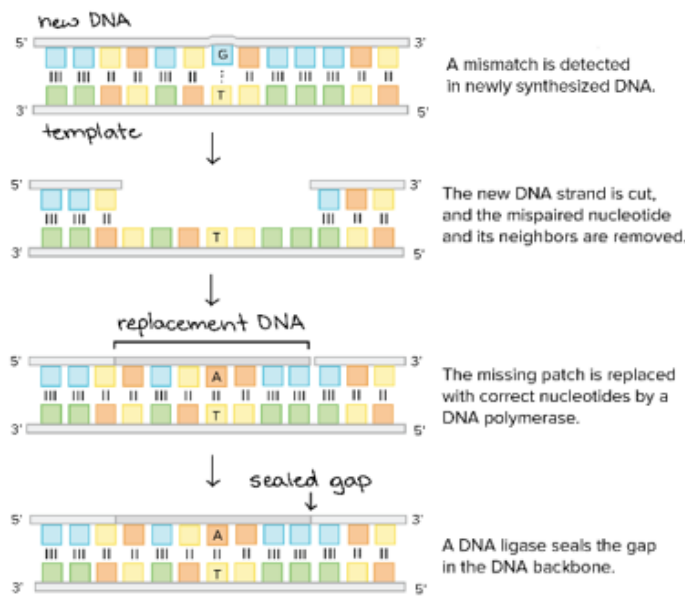

Figure 2: Illustrates DNA damage repair mechanism [1].

\section{Novel technique to repair damaged DNA}

DNA Mutations caused by UV radiations, tobacco smoke also causes damage to DNA. In the study researchers examined more than 20 million DNA mutations from 1,161 tumors across 14 cancer types. Experts found that many cancer types such as skin cancers, the number of alterations was particular high in regions of the genome called as "gene promoters". Significantly, these DNA sequences control how genes are stated which in turn determine cell type and their function.

Scientist presented that the numbers of DNA alterations are significantly increased in gene boosters because the proteins that bind DNA to control gene expression block one of the cell repair system is responsible for repairing damaged DNA. This system is known as nucleotide excision repair (NER) and is one of the number of DNR repair mechanisms that occurs in human cells and the only one capable of repairing damage from UV light.

This study was conducted by the team members and lead Dr. Jason Wong from Bioinformatics and Integrative Genomics at UNSW's Lowy Cancer Research Centre [4,5].

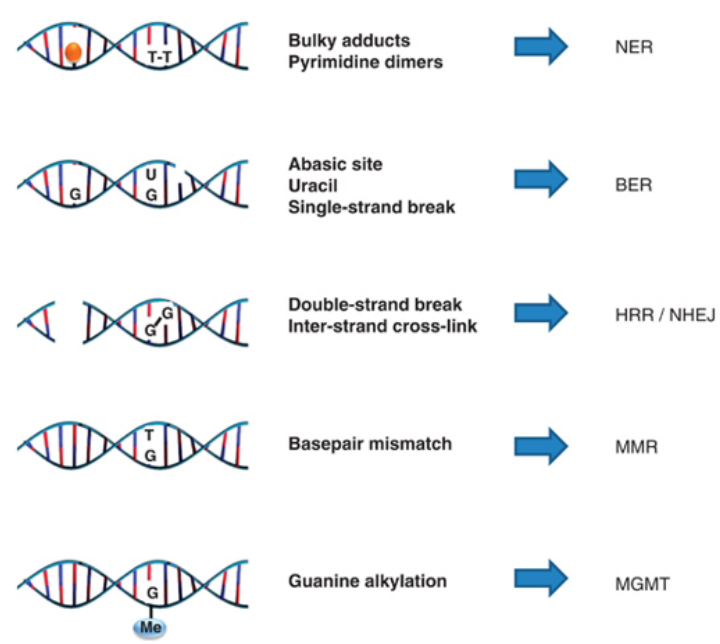

Figure 3: Illustrates Mechanisms of DNA repair. Different classes of DNA damage and the cellular mechanisms involved in repairing these. NER, nucleotide excision repair; BER, base excision repair; HRR, homologous recombination repair; NHEJ, non-homologous end joining; MMR, mismatch repair; and MGMT, 06-methyl-guanine-DNA methyltransferase [6].

\section{Scientists confirm DNA repair}

Employing advanced DNA sequencing technique to plan and analyze DNA damage, researchers validated the functions in bacterial cells of two important excision repair proteins that is Mfd and UvrD. To examine excision repair cells, researchers created a novel method, XR-seq, which allows examiners to separate and sequence to small lengths of adduct-damaged DNA that are snipped from the genome during excision repair process. Although, known that sequences of these DNA snippets allows their locations in the genome to map precisely. They used this method to generate a UV repair map of the human genome, later they used XR-seq technique to generate the damage and repair maps of anticancer cisplatin drug for entire human genome.

In un-sticker: Mfd - scientist used XR-seq to map UV-induced damaged in E.coli bacteria cells, experts found clear evidence of transcription -coupled repair in normal cells, but not in cells that lack Mfd, thus approving the protein's role in the process.

In unwinder: UvrD - scientist presented that the absence of UvrD, the excised piece of DNA remains bound to the chromosomal DNA, making it hard for cellular waste-disposal enzymes to chop it 
up. At that same time, the repair proteins that excised the strand tend to remain trapped to it and are kept moving on to excise other parts of damaged DNA. UvrD's main function is to unwind these damaged and discarded strands from chromosomal DNA, so that they can be discarded as quickly as possible and associated repair proteins can go on to catalyze additional rounds of repair $[7,8]$.

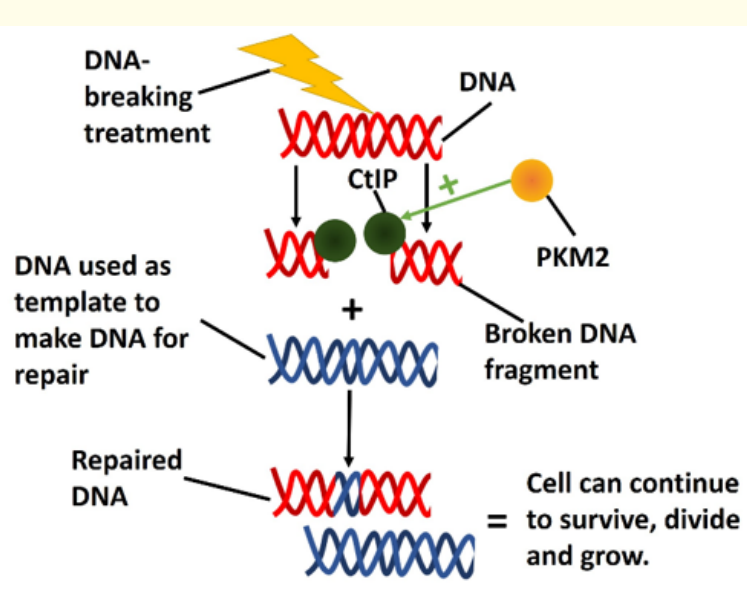

Figure 4: Illustrates DNA damage repair mechanism [9].

\section{Repairing faulty genes to cure diseases}

A new and novel method of genome editing technique known as CRISPR allows researchers to clip a specific DNA sequence and replace it with a new one, contributing the potential to cure diseases that are caused by defective genes.

Find and replace - The CRISPR system depends on cellular machinery that bacteria use to defend themselves from viral infection. Scientists had previously used this system to create gene editing complexes composed of a DNA cutting enzyme called Cas9 and short RNA that guides the enzyme to a specific area of the genome addressing Cas 9 where to make its cut.

When Cas9 and the short guide RNA targeting a disease gene are delivered directly to the cells, a specific cut is made in the genome and the cells. DNA repair processes glue the cut back together, often deleting a small percentage of the genome. Nevertheless, if a corrected copy of gene is also delivered when the cut is made. DNA repair can lead to correction of the diseases gene, permanently repairing the genome.
Scientists also wanted to boost the percentage of cells that had faulty gene replaced. In the previous work, about one in 250 liver cells were repaired, which was enough to successfully treat tyrosinemia. Nevertheless, for many other diseases, higher percentage of repair would be required to provide a therapeutic effect $[10,11]$.

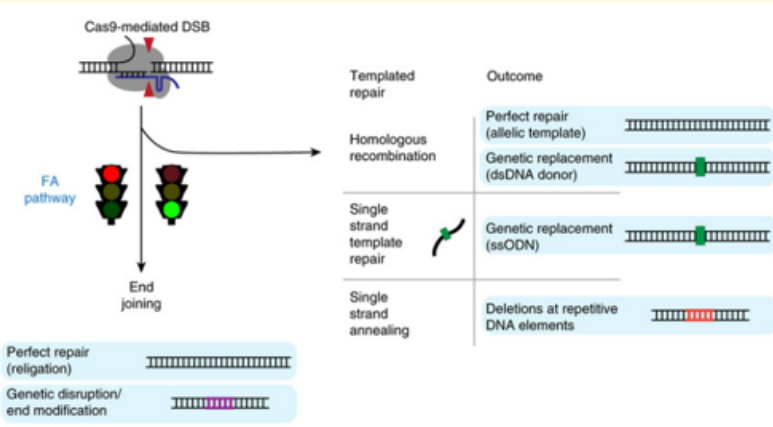

Figure 5: Illustrates The Fanconi anemia pathway acts as a traffic signal to route repair of double-stranded breaks through two different possible processes: end-joining, which requires no DNA template, and repair that requires a DNA template. As a result, only cells with an active Fanconi anemia DNA repair pathway can repair double-strand DNA breaks by homologous recombination, single-strand template repair or single-strand annealing. Jacob Corn lab, UC Berkeley [12].

\section{Repairing DNA: structure of MRE 11 protein bound}

Repairing breaks in two strands of the DNA double helix is very crucial for evading cancer. A molecular machine called MRN complex is liable for finding and signaling double-strand breaks (DSBs), then launching error-free method of DNA repair called homologous recombination. It is applicable in humans and as well as other organisms.

The fundamental part of MRN complex is the protein Mre11 (M stands for Mre11, R for Rad50 protein and N for Nbs1 protein). Without decent prototypes of how Mre11 interacts with DNA based on validations from high-resolution images, it is almost impossible to know how Mre11 recognizes the ends of broken DNA. Tainer and his team members resolved these issues by interpreting the images of the stand-alone structure of Mre11 to study Mre11 bound to DNA during the first steps of DSB repair. 
Homologous recombination is important not only for error free repair of DSBs but also for targeting genes to specific locations in the genome. It is a valuable method in cell biology and promising technique for gene therapy and other emerging medical interventions $[13,14]$.

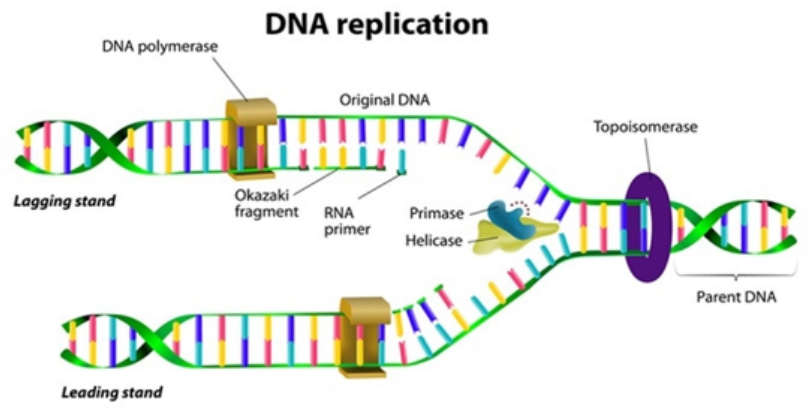

Figure 6: IIlustrates DNA replication [15].

\section{Discussion}

According to author's opinion it will give brief insight of various techniques and methods that can be not only be used in clinics but also various techniques can be formulated based on this review.

Because of its complexity and various methods, it will take more time to get more sophisticated mechanism and artificial methods to repair DNA.

\section{Conclusion}

To conclude there are many other various types of methods and techniques that is useful to repair DNA. This study of DNA gives a brief idea on DNA repairing and its mechanism. There are other ways to repair DNA using external ways.

\section{Conflicts of Interest}

There are no conflict of interest as per Author's point of view.

\section{Bibliography}

1. Khan Academy. DNA proofreading and repair (2019).

2. Wikipedia. DNA repair. In Wikipedia, the free encyclopedia (2019).

3. Public Library of Science. "New Mechanism Discovered for DNA Recombination and Repair". ScienceDaily (2019).
4. Dilmi Perera., et al. "Differential DNA repair underlies mutation hotspots at active promoters in cancer genomes". Nature 532.7598 (2016): 259.

5. University of New South Wales. "Repairing DNA damage in the human body". ScienceDaily (2019).

6. Lønning PE and Knappskog S. "Mapping genetic alterations causing chemoresistance in cancer: identifying the roads by tracking the drivers". Oncogene 32.46 (2013): 5315-5330.

7. Ogun Adebali., et al. "Genome-wide transcription-coupled repair in Escherichia coli is mediated by the Mfd translocase". Proceedings of the National Academy of Sciences (2017): 201700230.

8. University of North Carolina Health Care. "How life survives: Researchers confirm basic mechanism of DNA repair: Findings come from an advanced DNA sequencing application". ScienceDaily (2019).

9. Hawke A. How stopping cells from repairing DNA could help cure cancer (2019).

10. Hao Yin., et al. "Therapeutic genome editing by combined viral and non-viral delivery of CRISPR system components in vivo". Nature Biotechnology 34.3 (2016): 328-333.

11. Massachusetts Institute of Technology. "Curing disease by repairing faulty genes". ScienceDaily (2019).

12. Sanders R. DNA repair after CRISPR cutting not at all what people thought (2019).

13. Williams., et al. "Mre11 Dimers Coordinate DNA End Bridging and Nuclease Processing in Double-Strand-Break Repair". Cell 135.1 (2008): 97-109.

14. DOE/Lawrence Berkeley National Laboratory. "DNA Repair: Structure of The Mre11 Protein Bound To DNA". ScienceDaily (2008).

15. Smith Y. DNA Replication and Repair (2019).

\section{Volume 3 Issue 8 August 2019 \\ (C) All rights are reserved by Manu Mitra.}

